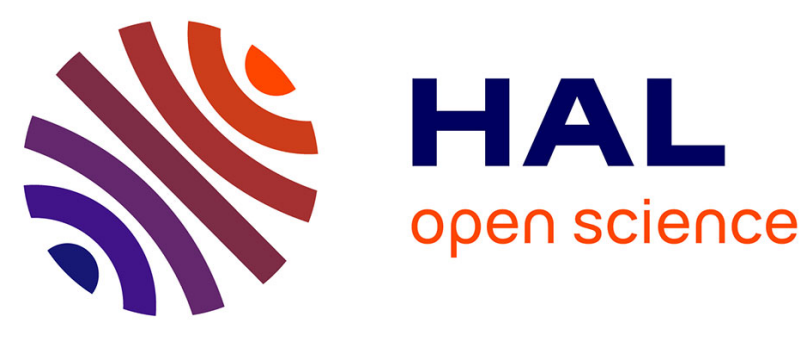

\title{
Sur un espace intellectuel
}

François Durand-Dastès

\section{To cite this version:}

François Durand-Dastès. Sur un espace intellectuel. Espace Géographique, 1989, 18 (2), pp.86-89. 10.3406/spgeo.1989.2848. hal-02459205

\section{HAL Id: hal-02459205 https://hal.science/hal-02459205}

Submitted on 29 Jan 2020

HAL is a multi-disciplinary open access archive for the deposit and dissemination of scientific research documents, whether they are published or not. The documents may come from teaching and research institutions in France or abroad, or from public or private research centers.
L'archive ouverte pluridisciplinaire HAL, est destinée au dépôt et à la diffusion de documents scientifiques de niveau recherche, publiés ou non, émanant des établissements d'enseignement et de recherche français ou étrangers, des laboratoires publics ou privés. 


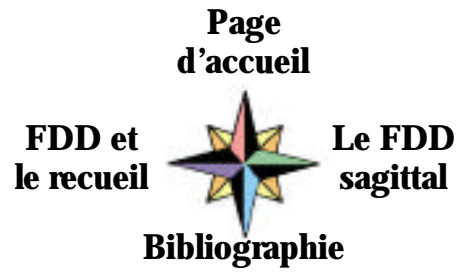

\section{Sur un espace intellectuel}

L'annonce d'une révision péniodique des programmes de l'enseignement secondaire a donc provoqué récemment une floraison de débats et de manifestes; de vieux démons se sont réveillés, que l'on aurait pu laisser domir, mais il s'est aussi posé de bonnes questions, tant en ce qui conceme la structure de la géographie qu'au sujet des choix à faire pour les élèves de l'enseignement secondaire. Les géographes ont été invités à des examens de conscience - on devrait plutôt dire des " examens d'intellect ", puisque nulle dimension morale n'est présente. Jouons le jeu, puisqu'on nous invite à le faire, en assumant le risque de la partialité et de la subjectivité.

\section{Un noyau dur aux limites floues}

Toute discipline a le droit de se définir à partir d'un certain nombre d'objets d'étude, de priorités, de questions de base, de points de vue privilégiés, qui en constituent le « noyau dur ». Dans cette perspective, on avancera ici que la géographie place au premier plan l'organisation de l'espace, son fonctionnement, qu'elle cherche à expliquer sa diversité ou plutôt sa diversification, aux différentes échelles, qu'elle pose comme prioritaire la question de la localisation (pourquoi est-ce là et pas ailleurs ?) ; qu'elle part de l'espace pour interroger le réel, et singulièrement la société, en rapport avec son support matériel, hénité à la fois de l'action des générations passées et des forces de la nature.

Pour étudier cet espace, objet de tant de convergences et d'interactions, les géographes ont rencontré, voire constitué d'autres disciplines, vers lesquelles ils ont beaucoup exporté et auxquelles ils ont beaucoup emprunté, et qui leur ont beaucoup appris.

Pourquoi ne pas reconnaître qu'il s'est formé, aux marges du "noyau dur" de la géographie, des pratiques et des savoirs largement autonomes, comme la géomorphologie ou la climatologie mais aussi, car le monde "physique " n'est pas seul en cause, des sociologies plus ou moins spatialisées (" urbaines » ou " nurales »), de l'économie spatiale plus ou moins modélisée? Autonomie ne veut pas dire indépendance, c'est bien connu, encore moins indifférence ou hostilité.

Vers ces savoirs et ces pratiques "de bordure", la géographie exporte beaucoup. D'abord des individus. On peut devenir excellent géomorphologue, sociologue urbain, économètre modélisateur, à partir d'une formation de géographe. En d'autres temes, bien des géographes travaillent hors du noyau dur de leur discipline, et peuvent même cesser de se considérer comme géographes (ou d'abord comme géographes, car il leur reste toujours quelque chose de leur origine). L'essentiel n'est-il pas qu'ils fassent du travail intéressant pour eux comme pour la communauté scientifique ? Cette circulation vers les marges peut même comporter de féconds allers et retours ; on peut même penser qu'il n'est pas forcément nécessaire ou souhaitable de se situer sans intemuption dans le noyau dur, mais c'est là matière à discussion. Ce qui vient d'être dit des individus est aussi valable pour les méthodes, les concepts et les intermogations. 
Ainsi peut se constituer l'image d'une discipline qui a son domaine propre, un centre fort et, aux limites, des excroissances (pour reprendre une expression de G. et Ph. Pinchemel), des savoirs plus spécialisés et limités, largement pratiqués par des géographes de formation. Ils dessinent en quelque sorte, autour du noyau dur, une première auréole. Une seconde auréole serait alors constituée par des disciplines plus éloignées, plus autonomes, dont les spécialistes ont reçu une formation propre. Entre les différentes parties de l'espace intellectuel ainsi défini, les échanges de personnes et d'informations ne peuvent qu'être féconds. Mais il n'est pas pour autant nécessaire de vouloir à toute force intégrer à la géographie des branches qui s'en sont éloignées ou détachées, tout en restant en contact avec elle ; on peut penser que certaines d'entre elles ont une place légitime dans des instituts ou des départements de géographie, bien qu'il y ait, là aussi, matière à débat.

Dans cette perspective, le noyau central de la géographie offre l'image d'une discipline carrefour, voire, nisquons le mot, " de synthèse ". La notion déplaît à bien des gens. Et il est bien vrai qu'elle ne saurait servir à définir la géographie, dans son originalité, comme on a souvent essayé de le faire, quand ce ne serait que dans la mesure où ce caractère n'est en rien spécifique de la géographie. Toute discipline en effet se trouve plus ou moins au carrefour de plusieurs autres, et développe ses propres excroissances tout en élaborant sa propre synthèse, en fonction de ses intermogations centrales. Ce qui vient d'être dit de la géographie pourrait aussi bien l'être de la sociologie, de l'histoire, de la physique ou de la biologie, et, a fortioni, de la philosophie ou de la médecine. A peine pourait-on avancer, prudemment, que ces deux demières disciplines sont, avec la géographie, celles qui ont par nature, les contacts exténieurs les plus nombreux et les plus divers.

Il amive que, dans le temps, les intermogations centrales soient réorientées, situées dans de nouvelles perspectives, que les échanges interdisciplinaires soient reconsidérés ; bref, qu'il y ait des révisions et des remises en cause. Certains les ressentent comme des crises d'identité à propos de leur discipline favorite. Il n'y a là rien ni de spécifique ni de tragique. Les nouveaux savoirs, les nouvelles méthodes, voire les changements de paradigme (moins fréquents qu'on ne le dit parfois) provoquent des changements qui sont signes de vitalité et d'activité, plus stimulants qu'accablants.

Et il en va de même dans toutes les branches du savoir ; il suffit pour s'en convaincre de feuilleter les revues spécialisées, les articles d'encyclopédies, les bilans présentés à diverses occasions par toutes les disciplines. Ni la diversité, ni les discussions, ni les problèmes de définition ne sont spécifiques de la géographie ; et pas plus pour celle-ci que pour les autres n'y a-t-il lieu de s'en affliger.

On a pu avancer que le débat sur les rapports entre l'action humaine et le milieu naturel serait un caractère particulièrement douloureux de la géographie ; et il est bien vrai qu'il alimente des polémiques, souvent acenbes. Ne pourrait-on envisager utilement à ce propos de changer un peu de perspective, en essayant en quelque sorte de déplacer les lignes de clivage ? En effet, l'action humaine, celle de chaque nouvelle génération, et donc aussi des sociétés contemporaines, se trouve confrontée à un espace diversifié, dans une certaine mesure solidifié, que j'ai il y a quelque temps proposé d'appeler « l'espace reçu ». Celui-ci exerce une pesanteur sur les sociétés. On peut admettre, suivant une expression devenue classique, que celles-ci produisent de l'espace ; mais elles ne le produisent pas à partir de rien, elles n'écrivent pas sur une page vienge. Il s'établit toute 
une sénie d'interactions entre les sociétés, leurs pouvoirs, leurs techniques, et cet espace reçu. Or celui-ci est le résultat à la fois de facteurs naturels et de l'action des générations passées. On peut se demander si la ligne de clivage essentielle passe bien entre les héritages de la nature et ceux du passé de l'humanité. Peut-être serait-il plus utile de prendre en compte les forces en jeu et les temps de réaction. Il existe toute une gradation de ces deux points de vue. Les forces qui ont mis en place les grandes masses continentales et les volumes du relief sont sans commune mesure avec les forces humaines et la durée de la présence de l'homo sapiens; le dessin et le volume des continents occupent à cet égard une place particulière dans l'espace reçu. Mais un réseau urbain, certaines constructions politiques, ont une durée, un temps de réaction, du même ordre de grandeur que ceux qui caractérisent les formations végétales, les sols, les microclimats ; les forces qui gouvement les uns et les autres et permettent de les modifier sont aussi du même ordre de grandeur. A un stade intermédiaire, les macro-climats et les grandes civilisations sont aussi comparables, des deux points de vue des forces et des temps.

La liberté des sociétés humaines, même les plus prométhéennes, est limitée par l'espace reçu, ou encore par les " contraintes inscrites » dans l'espace. Les forces et les échelles de temps qui ont inscrit ces contraintes sont peut-être plus importantes à prendre en compte de façon prionitaire que le clivage entre ce qui revient à la nature ou à l'histoire humaine. Aussi bien, la distinction est souvent difficile.

\section{Des choix didactiques}

Pour des raisons en partie historiques, la géographie, ou plutôt les géographes, se sont trouvés chargés dans l'enseignement secondaire de la très belle tâche de présenter le Monde aux jeunes Français, de tenir un discours scientifique sur « l'ailleurs » pour ceux qui sont « ici ». On aurait envie d'affimer que la géographie est bien placée pour le faire, mais qu'en raison de certaines contraintes elle ne le fait pas aussi bien qu'on pourrait le souhaiter, et qu'enfin on pourrait imaginer qu'elle fasse autre chose, ou aussi autre chose.

Pour parler du Monde à ceux qui l'habitent, la géographie est sans doute irremplaçable, à cause de sa vocation globalisante, de la complexité des systèmes logiques qu'elle met en ouvre dans l'explication. Les autres disciplines ont pour la plupart recours à des schémas explicatifs plus simples, qui ont parfois l'avantage d'être plus rigoureux, mais l'inconvénient d'être moins complets. Pour nous en tenir à un exemple, il est vrai que les économistes, notamment les économistes spatiaux de "l'école de la régulation ", ont apporté des éléments très importants sur la différenciation des espaces, tant à l'échelle intemationale qu'au niveau intra-national. Ils ont montré que le fonctionnement des systèmes mondial et nationaux impliquent des divisions spatiales du travail, une distribution des rôles entre des espaces. Mais leurs travaux restent peu satisfaisants pour expliquer la répartition des rôles. Ils savent dire pourquoi l'espace est différencié, beaucoup moins bien pourquoi on observe une certaine différenciation et pas une autre, ce qui est pourtant fondamental. En d'autres temes, ils ne vont pas assez loin dans l'étude de la question de localisation, alors qu'elle est très importante, et très intéressante. On ne peut s'empêcher de trouver que le géographe est capable d'aller plus loin dans le domaine du "pourquoi ? ", et pose ainsi des problèmes qui ont une grande valeur culturelle. 
Cependant, la situation est loin d'être entièrement satisfaisante ; on peut imaginer d'expliquer cela par un certain nombre de contraintes qui peuvent provenir du quasimonopole de la géographie dans la tâche qui lui est ainsi impartie, ou, si l'on préfère, de son isolement en la matière.

La nécessité de présenter un tableau exhaustif conduit, surtout dans les petites classes, à une certaine rapidité, qui incite souvent le géographe à privilégier le constat, à apporter une explication, et une seule, dans le même temps où il décrit un fait ; il consacre peu de place, de ce fait, à l'exposé des altematives dans le domaine de l'explication, aux questions ouvertes, voire au mystère et à l'étonnement. C'est sans doute cela qui est la cause principale du côté « bonasse » de la " géographie des professeurs », dénoncé parY. Lacoste.

Dans les grandes classes, la même contrainte conduit à privilégier l'étude de certains espaces, ceux qui sont "importants ", c'est-à-dire la France et les grandes puissances économiques. De ce fait, les élèves sont plus ou moins privés de références à d'autres espaces, dont l'étude aurait pour eux une grande valeur culturelle. L'organisation de l'espace, ses rapports avec la société, soulèvent des questions aussi intéressantes et formatrices dans des régions exclues du programme, ou tout au moins marginalisées, qu'en URSS, aux Etats-Unis ou au Japon. C'est toujours pour la même raison que les problèmes économiques sont privilégiés aux dépens des structures spatiales, et, du même coup, de bien des aspects des sociétés et des organisations politiques. J'ai entendu un jour une inspectrice pédagogique régionale dire qu'elle conseillait aux professeurs qui dépendaient d'elle de parler " plutôt du cours du dollar que de la Califomie ». De même, on peut lire dans un livre de poche publié en 1985 sous l'égide du Ministère de l'Education Nationale le programme suivant pour la classe de cinquième : " Les élèves découvrent l'Afrique, l'Asie, l'Amérique latine. Ils comprennent les problèmes que pose la mise en valeur des ressources de la Terre pour les hommes d'aujourd'hui, c'est-à-dire la notion de développement ». Et, plus loin : "L'Asie. Le riz et les civilisations de l'Asie des Moussons. Le développement en Inde, en Chine, au Japon ». Certes, les questions ainsi proposées ne sont pas des moindres. Mais l'espace, les sociétés et les civilisations indiennes et chinoises ne peuvent-elles pas être évoquées en d'autres termes ? Les limitations qui en fonction de ce libellé, ont été suggérées aux directeurs de collections de manuels, sont-elles raisonnables et acceptables?

Au risque de déplaire, on pourrait avancer que des enseignements plus spécialisés sur l'économie mondiale libéreraient du temps pour l'évocation de problèmes plus spécifiquement géographiques, pour l'étude et l'explication de la différenciation spatiale, avec toute sa richesse et toute sa valeur formatrice, tant du point de vue intellectuel que du point de vue civique. Peut-être la géographie gagnerait-elle à être davantage ellemême, si certaines des tâches qu'elle assume étaient prises en charge différemment, peut-être par d'autres. La question ménite d'être posée.

Enfin, il n'est pas évident que la seule présence de la géographie doive être la présentation du Monde telle qu'elle est pratiquée. On pourrait imaginer une option assez différente, qui donnerait plus de place à une étude systématique et organisée des processus de différenciation spatiale, Certes, la " géographie générale » est présente dans les programmes, et elle donne lieu à beaucoup de bon travail. Mais on peut trouver qu'elle reste timide et très marquée par le clivage physique-humain ; sans doute parce qu'elle est largement conçue comme une préparation à la " géographie régionale ». Cette timidité se marque par une certaine réticence en matière conceptuelle. Elle se prive 
encore largement du recours au raisonnement déductif, à la modélisation. On préfère encore les fameux " exemples bien choisis » aux types idéaux au sens de M. Weber, ou aux modèles. Les simulations, les jeux spatiaux, sont encore largement absents de l'enseignement français. Il semble qu'il en aille tout autrement dans des pays voisins, outre-Manche par exemple. Sans doute y a-t-il toute une série de possibilités dans ce domaine (et, incidemment, matière à une utilisation de l'ordinateur plus intéressante que celle qui est encore pratiquée pour le moment dans la plupart des cas).

Il ne faut pas se dissimuler que ces perspectives nouvelles conduiraient sans doute à des choix, dont certains pourraient être ressentis comme douloureux. Mais le dossier d'une géographie plus conceptuelle, plus " générale ", et, disons le mot, plus " abstraite ", peut être plaidé. Après tout, les élèves des grandes classes sont invités à s'initier à l'abstraction et à la généralisation en mathématiques, en philosophie, en physique. Pourquoi la géographie devrait-elle rester exclue de cette formation intellectuelle ?

L'E space Géographique, 1989, n 2, p. 86-89. 